\title{
A Longitudinal Study of Clinical Benefits with Implementation of the Deep Inspiration Breath-Hold Technique in Post-Operative Radiotherapy for Left-Sided Breast Cancer
}

\author{
Chunhui Han*, Nayana Vora, Sean Zhang, An Liu, Jeffrey Y. C. Wong \\ Department of Radiation Oncology, City of Hope National Medical Center, Duarte, CA, USA \\ Email: *chan@coh.org
}

How to cite this paper: Han, C.H., Vora, N., Zhang, S., Liu, A. and Wong, J.Y.C. (2019) A Longitudinal Study of Clinical Benefits with Implementation of the Deep Inspiration Breath-Hold Technique in Post-Operative Radiotherapy for Left-Sided Breast Cancer. International Journal of Medical Physics, Clinical Engineering and Radiation Oncology, 8, 151-162. https://doi.org/10.4236/ijmpcero.2019.83014

Received: June 10, 2019

Accepted: August 25, 2019

Published: August 28, 2019

Copyright $\odot 2019$ by author(s) and Scientific Research Publishing Inc. This work is licensed under the Creative Commons Attribution International License (CC BY 4.0).

http://creativecommons.org/licenses/by/4.0/

\begin{abstract}
Purpose/Objectives: We aimed to report clinical effects on critical organ dose and cardiac toxicity from implementation of the deep inspiration breath-hold (DIBH) technique in post-operative extern-beam radiotherapy of patients with left-sided breast cancer, using longitudinal clinical data. Materials/Methods: We retrieved three groups of patients who received post-operative radiotherapy of left-sided breast cancer in our institution in recent years: Groups A and B consist of patients whose treatment did not include internal mammary nodes (IMN) and who were treated with the free breathing technique and with the DIBH technique, respectively, and Group C consists of patients whose radiotherapy included internal mammary nodes with the DIBH technique. Dose parameters for the heart and left lung were retrieved from the treatment plans. Radiation-induced cardiac risks were estimated using existing risk models. Results: The average heart dose was $2.65 \pm 0.98$ Gy, $1.10 \pm$ $0.29 \mathrm{~Gy}$, and $1.26 \pm 0.25 \mathrm{~Gy}$ in Groups A, B, and C, respectively. The average heart volumes receiving at least $25 \mathrm{~Gy}$ were $7.10 \pm 9.79 \mathrm{cc}$ in Group A, $0.07 \pm$ $0.22 \mathrm{cc}$ in Group B, and $0.03 \pm 0.08 \mathrm{cc}$ in Group C. On average, the excessive risk of having ischemic heart disease was estimated to be $19.6 \%, 8.1 \%$, and 9.3\% in Groups A, B, and C, respectively. The mean left lung doses were 5.73 $\pm 1.86 \mathrm{~Gy}, 5.93 \pm 1.55 \mathrm{~Gy}$, and $9.13 \pm 1.57 \mathrm{~Gy}$ in Groups A, B, and C, respectively. Conclusion: Implementation of the DIBH technique significantly lowered heart dose and decreased the ischemic heart disease risk in patients receiving post-operative radiotherapy for left-sided breast cancer, without significant increase in left lung dose.
\end{abstract}

\section{Keywords}

Breast Cancer, Heart Toxicity, Breath Hold, Extern-Beam Radiotherapy 


\section{Introduction}

Breast cancer is the most commonly diagnosed cancer in women in the United States with 268,600 newly diagnosed breast cancer cases expected in 2019 [1]. Post-operative radiation therapy (RT) is an important adjuvant treatment modality for lowering cancer recurrence rate [2] [3]. For early-stage breast cancer patients that meet certain selection criteria, partial breast irradiation could be a feasible option with good treatment outcome [4]. For most patients, post-lumpectomy whole breast radiation or post-mastectomy chestwall irradiation, with or without irradiation of regional lymph nodes, is the current standard of care for lowering cancer recurrence rate [2] [5].

Despite technological development in intensity-modulated radiotherapy (IMRT) techniques, three-dimensional conformal radiotherapy (3D-CRT) techniques using opposed tangential fields are still widely used. In post-operative external beam irradiation of the left-sided breast cancer patients, potential side effects include acute and chronic toxicities to the lung, ribs, heart, skin, as well as increased risks of radiation-induced secondary cancer occurrence in the thoracic region [6] [7] [8] [9] [10]. Heart and lung toxicities could lead to sever long-term morbidity and mortality [6]. Recent studies have shown that the risk of long-term ischemic heart diseases increases with increasing radiation dose to the heart [11]. Given good survival expectations for breast cancer patients, special attention should be paid to the heart and lung dose during post-operative irradiation to minimize long-term cardiovascular and pulmonary morbidity and mortality rates.

One method to reduce heart dose in post-operative irradiation of left-sided breast cancer patients is the deep-inspiration breath-hold (DIBH) technique, in which the patient takes a deep breath to elevate the chestwall from the heart. Most published studies on the DIBH technique performed pre-clinical dosimetric comparison with different treatment techniques with a limited number of cases [12] [13] [14] [15]. To evaluate clinically achievable benefits with the DIBH technique, we carried out this study to report our clinical experience with the DIBH technique based on large-scale longitudinal clinical data over a six-year period. The goals of this study are: 1) to evaluate clinically achievable reduction of heart dose with the implementation of the DIBH technique for post-operative left-sided breast cancer patients; 2) to estimate the reduction of ischemic heart disease risks with the DIBH technique; and 3) to evaluate dosimetric effect on the inflated left lung volume with the DIBH technique.

\section{Methods and Materials}

In 2013, our institution clinically implemented the DIBH technique in external beam RT of left-sided breast cancer patients. In this study, we retrospectively retrieved the following three groups of patients from the clinical database for comparison. The first two groups of patients did not receive external-beam RT to the internal mammary lymph nodes (IMN): Group A consists of patients who 
received post-operative external-beam RT to the left breast or chestwall with normal breathing in the three years prior to the clinical implementation of the DIBH technique, and Group B consists of patients who received post-operative external-beam RT to the left breast or chestwall using the DIBH technique in the three years after the clinical implementation of the DIBH technique. A treatment plan would be excluded from either Group A or Group B if the IMN was intentionally included as target in treatment planning. The third group of patients, Group C, consists of patients who received post-operative external-beam RT to the left breast or chestwall using the DIBH technique in the three years after the clinical implementation of the DIBH technique and whose treatment plans used widetangential photon fields to cover the IMN in addition to the left breast or chestwall. The list of candidate patients was first retrieved from the clinical database by using an in-house data mining software application. Then each treatment plan was reviewed individually for eligibility to be included in this study: a treatment plan would be excluded from this study if the heart or the left lung was not contoured at the time of treatment planning. At the end of the case selection process, 104 and 105 patients were included in Groups A and B respectively, and 22 patients were included in Group C. Table 1 lists characteristics of the three groups of patients.

All the patients in Group A were treated without using the DIBH technique. Before treatment planning, each patient received a three-dimensional planning CT simulation (3DCT) (Siemens AcQSim CT, Siemens AG, Munich, Germany) in a supine position. With the patient on the CT simulation couch, the radiation oncologist placed radiopaque wires to denote the borders of the intended treatment region in the superior, inferior, lateral, and medial directions. The patient was simulated with both arms stretched over the head, holding two handles on a wing board superior to the head. An incline board was placed under the patient so that the chestwall was approximately parallel to the CT couch. Skin marks were drawn on the patient to denote room laser incidence on the patient skin. The CT simulation was done in a helical mode, using a 3-mm slice thickness and spacing. During CT simulation as well as during treatments, patients were instructed to maintain shallow breaths. The CT simulation images were then transferred to a treatment planning system (TPS) (Eclipse, Varian Medical Systems,

Table 1. Characteristics of patients in each of the two patient groups used in this study.

\begin{tabular}{cccccc}
\hline Patient Group & Group A & Group B & Group C & \multicolumn{2}{c}{ p-value } \\
\cline { 5 - 6 } & & & & A - B & A - C \\
\hline Number of patients & 104 & 105 & 22 & N/A & N/A \\
$\begin{array}{c}\text { Number of patients at } \\
\text { each prescription dose }\end{array}$ & $68: 50.4 \mathrm{~Gy}$ & $79: 50.4 \mathrm{~Gy}$ & $20: 50.4 \mathrm{~Gy}$ & N/A & N/A \\
$\begin{array}{c}\text { Average age } \\
\text { (range)/years old }\end{array}$ & $58 \pm 10$ & $58 \pm 11$ & $58 \pm 7$ & & 0.66 \\
\hline
\end{tabular}

The p-values are from unpaired t-tests between two patient groups. A - B: The p-value for t-test between Groups A and B; A - C: The p-value for t-test between Groups A and C. N/A: Not applicable. 
Palo Alto, CA), where structures including the heart and the lungs were delineated. The heart contour included pericardium but not the great vessels.

All the patients in both Groups B and C were treated using the DIBH technique. During the CT simulation, the patient had the same setup position as those in Group A. The same type of wing board and incline board were used to position the patient on the CT couch. A respiratory motion motoring system (Real-time Positioning Management (RPM) system, Varian Medical Systems, Palo Alto, CA) was used during simulation to record the breath-hold magnitude. A respiratory marker block was placed on the skin of the abdominal area, inferior to the xiphoid process, to monitor the magnitude of respiratory motion. Right before initiation of the CT scan, the patient was asked to take a deep breath and hold the breath for some time, while a 3DCT was taken in the thoracic region in a helical scan mode (GE PET/CT Optima PET/CT, General Electric, Boston, MA). The breath-hold magnitude during the CT simulation was monitored by the RPM system and recorded in the patient chart to guide treatment. The CT simulation images were then transferred to a treatment planning system for delineation of structures including the heart and the lungs.

For each patient in this study, a two-field or three-field three-dimensional conformal radiotherapy (3D-CRT) plan was generated using $6-\mathrm{MV}$ or $10-\mathrm{MV}$ photon beams on medical linacs. In a two-field treatment plan, two opposed tangential photon beams were used to deliver radiation to the left breast or the left chestwall. In a three-field treatment plan, one anterior supraclavicular field was used in addition to two tangential fields, with the field isocenter at the inferior edge of the supraclavicular field and at the superior edges of the tangential fields. The prescribed dose was either $50 \mathrm{~Gy}$ in 25 fractions or $50.4 \mathrm{~Gy}$ in 28 fractions. To achieve adequate dose homogeneity in the target region, either wedges or the field-in-field technique was used for the tangential fields. For both Groups $\mathrm{A}$ and $\mathrm{B}$ patients, the primary goal in treatment planning was to cover the entire left chestwall or left breast while minimizing dose to the left lung and the heart as much as possible. For Group $\mathrm{C}$ patients, wide tangential fields were used to cover the IMN. In all the treatment plans, the anisotropic analytical algorithm (AAA) was used for dose calculations with heterogeneity correction.

On the first day of treatment, each patient in Group A was set up on the treatment couch by first aligning room lasers to the skin marks on the patient and then shifting the couch to move the isocenter to the intended location as in the treatment plan. Portal images were then taken and compared to planning digitally reconstructed radiotherapy (DRR) images to verify the patient position. Patients were asked to maintain shallow breathing both during setup and during each treatment session. The same immobilization devices as used during the CT simulation were used for daily setup and treatment. Throughout the treatment course, portal images were taken every week and reviewed by the radiation oncologist.

All the patients in both Groups B and C were treated on a linac with respiratory monitoring and gating capabilities (TrueBeamlinac, Varian Medical Sys- 
tems, Palo Alto, CA). During daily treatments, a respiratory marker block was placed in the same abdominal area as during the CT simulation to measure respiratory motion by infrared cameras. During patient setup, the patient was first positioned by aligning room lasers to the skin marks on the patients and then shifting the couch to move the isocenter to the intended location as in the treatment plan. Then the patient was instructed to take a deep breath and hold the breath when portal images were taken. On the linac console, therapists set upper and lower limits to the breath-hold treatment window based on the recorded breath-hold magnitude during the CT simulation. Therapy beam was turned on for both portal imaging and dose delivery only when the breath-hold magnitude fell within the upper and lower limits of the preset breath-hold treatment window. If the patient could not hold the breath long enough during portal imaging or treatment, the beam would automatically stop when the breath-hold magnitude fell below the lower limit of the treatment window and would resume after the patient took another deep breath to move the breath-hold magnitude back to the treatment window. Portal images were taken every day for patients in Groups B and C, and then the images were reviewed by the radiation oncologist.

All the dosimetric data in this study were retrieved from the clinical database of the TPS. To facilitate data retrieval, an in-house data mining software application was written which extracted dosimetric data in the treatment plans for all the patients in this study. For the heart, the mean dose, median dose, and V25 (the heart volume receiving at least $25 \mathrm{~Gy}$ ) were extracted and analyzed. For the left lung, the mean dose, median dose, left lung volume, and left lung V20 (the left lung volume receiving at least $20 \mathrm{~Gy}$ ) were extracted and analyzed. Unpaired t-tests were used to compare dosimetric data between different patient groups.

\section{Results}

Table 2 lists statistics of dosimetric parameters for the heart in the treatment plans in each group. Unpaired t-tests showed statistically significant difference in the mean heart dose, median heart dose, and heart V25 between Groups A and $\mathrm{B}$ and between Groups $\mathrm{A}$ and $\mathrm{C}$, respectively. The average mean heart dose in Group B was $1.10 \pm 0.29 \mathrm{~Gy}$, which was $58.5 \%$ lower compared to the average mean heart dose of $2.65 \pm 0.98 \mathrm{~Gy}$ in Group A. The average mean heart dose in Group C was $1.26 \pm 0.25 \mathrm{~Gy}$, which was $52.5 \%$ lower compared to the average mean heart dose in Group A. Figure 1 shows normalized mean heart dose distribution in each patient group. In Group B, less than 5\% (5 cases) of the cases showed a mean heart dose higher than $1.5 \mathrm{~Gy}$; in comparison, over $77 \%$ (80 cases) of the cases in Group A had a mean heart dose higher than $2.0 \mathrm{~Gy}$. In all the Group B cases the heart V25 was no more than $0.25 \%$. In $85.7 \%$ (90 cases) of the cases in Group B, the heart V25 was less than $0.1 \mathrm{cc}$. For directly comparison, Figure 2(a) shows the average heart DVH for each patient group.

Based on results by Darby et al. [11], the increased risk of ischemic heart disease due to ionizing radiation was evaluated for each patient group, and the 
Table 2. Mean and standard deviations for dosimetric parameters of the heart and increase in ischemic heart disease risk in each patient group.

\begin{tabular}{ccccccc}
\hline \multirow{2}{*}{ Patient Group } & Group A & Group B & Group C & \multicolumn{3}{c}{ p-value } \\
\cline { 6 - 7 } & & & & $\mathrm{A}-\mathrm{B}$ & $\mathrm{A}-\mathrm{C}$ & $\mathrm{B}-\mathrm{C}$ \\
\hline \multirow{2}{*}{ Mean heart dose/Gy } & $2.65 \pm 0.98$ & $1.10 \pm 0.29$ & $1.26 \pm 0.25$ & & & \\
& $(0.95-6.49)$ & $(0.46-1.85)$ & $(0.89-1.81)$ & & $<0.01$ & 0.01 \\
Median heart dose/Gy & $1.60 \pm 0.43$ & $0.72 \pm 0.19$ & $0.85 \pm 0.24$ & & & \\
& $(0.53-3.00)$ & $(0.35-1.27)$ & $(0.56-1.54)$ & $<0.01$ & $<0.01$ & 0.03 \\
Heart V25/\% & $1.27 \pm 1.66$ & $0.01 \pm 0.04$ & $0.01 \pm 0.02$ & & & \\
& $(0.00-8.82)$ & $(0.00-0.25)$ & $(0.00-0.06)$ & $<0.01$ & $<0.01$ & 0.09 \\
Heart V25/cm ${ }^{3}$ & $7.10 \pm 9.79$ & $0.07 \pm 0.22$ & $0.03 \pm 0.08$ & & & \\
& $(0.00-52.00)$ & $(0.00-1.47)$ & $(0.00-0.30)$ & $<0.01$ & $<0.01$ & 0.11 \\
Increased Risk of Ischemic & $19.6 \pm 7.25$ & $8.1 \pm 2.15$ & $9.3 \pm 1.85$ & & & \\
heart disease/\% & $(7.03-40.03)$ & $(3.40-13.69)$ & $(6.59-13.39)$ & $<0.01$ & $<0.01$ & 0.01 \\
\hline
\end{tabular}

The $\mathrm{p}$-values are from unpaired $\mathrm{t}$-tests between two patient groups. $\mathrm{A}-\mathrm{B}$ : The $\mathrm{p}$-value for $\mathrm{t}$-test between Groups A and B; A - C: The p-value for t-test between Groups A and C; B - C: The p-value for t-test between Groups B and C.

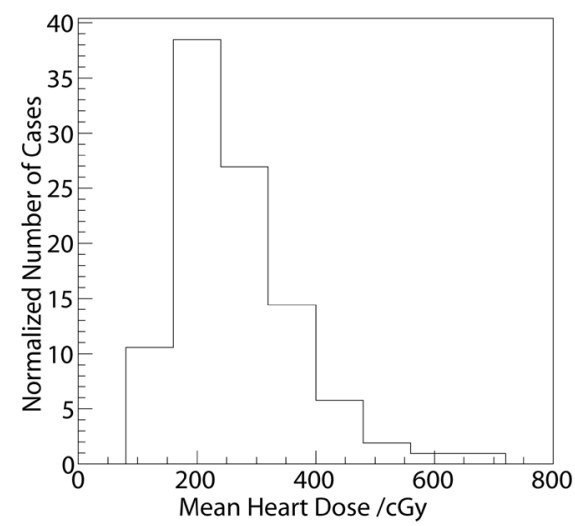

(a)

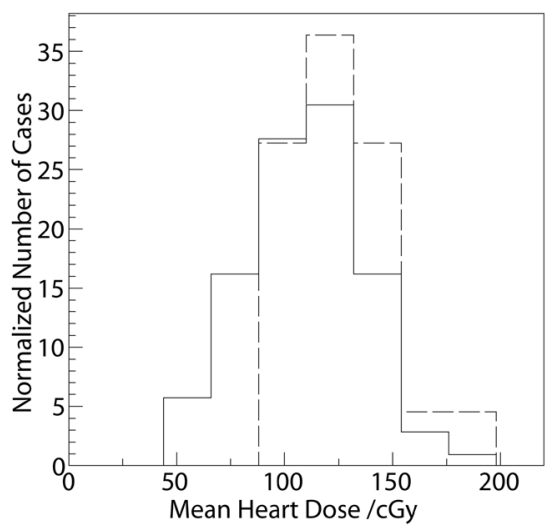

(b)

Figure 1. Normalized mean heart dose distributions for each group: (a) Group A; (b) Group B (solid lines) and Group C (dashed lines).

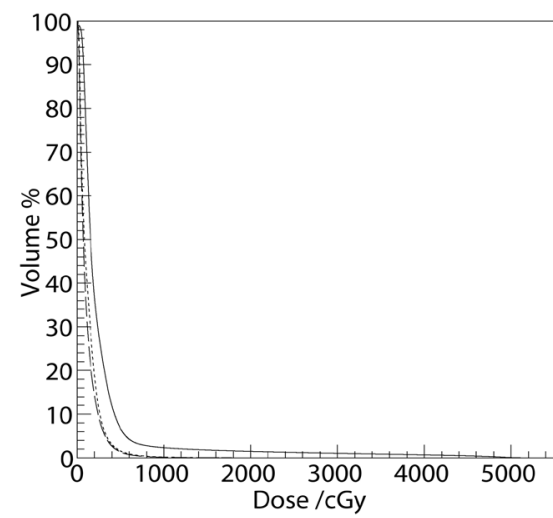

(a)

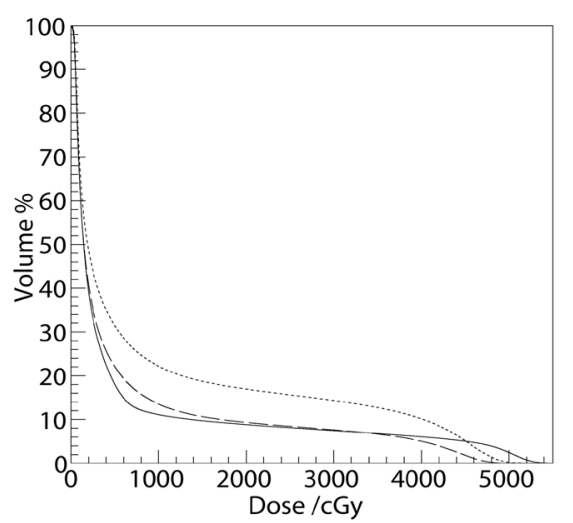

(b)

Figure 2. Average dose volume histograms (DVHs) for the heart and the left lung in Groups A, B, and C, respectively. (a) Average DVHs for the heart; (b) Average DVHs for the left lung. Group A: solid curves; Group B: dashed curves; Group C: dotted curves. 
results were listed in Table 2 . Based on the mean heart dose statistics, there was an average increased risk of $19.6 \%, 8.1 \%$, and $9.3 \%$ for patients in Groups A, B, and $C$, respectively, with the difference in risks being statistically significant between Groups A and B and between Groups A and C (two-tailed p-value $<0.01$ in unpaired t-tests). Compared to ischemic heart disease risks in Group A, there was a $58.5 \%$ reduction in risks in Group B when the DIBH technique was implemented, and a $52.6 \%$ reduction in risks in Group $\mathrm{C}$ even with the use of wide tangential fields.

Table 3 lists statistics of dosimetric parameters for the left lung in each patient group. Figure 2(b) plots the average left lung DVH in each patient group. When the data for both Groups $\mathrm{B}$ and $\mathrm{C}$ were combined, the average left lung volume for patients using the DIBH technique was $1851 \pm 337 \mathrm{cc}$, indicating an increase of $71.7 \%$ compared to the average left lung volume of $1078 \pm 290 \mathrm{cc}$ in Group A. Figure 3(a) shows normalized left lung volume distributions in each patient

Table 3. Mean and standard deviations of left lung volume and dosimetric parameters in each patient group in this study.

\begin{tabular}{|c|c|c|c|c|c|}
\hline \multirow{2}{*}{ Patient Group } & \multirow{2}{*}{ Group A } & \multirow{2}{*}{ Group B } & \multirow{2}{*}{ Group C } & \multicolumn{2}{|c|}{$\mathrm{p}$-value } \\
\hline & & & & $A-B$ & $A-C$ \\
\hline Left lung volume/cc & $\begin{array}{c}1078 \pm 290 \\
(633-2073)\end{array}$ & $\begin{array}{c}1834 \pm 345 \\
(852-2742)\end{array}$ & $\begin{array}{c}1889 \pm 304 \\
(1157-2494)\end{array}$ & $<0.01$ & $<0.01$ \\
\hline Mean left lung dose/Gy & $\begin{array}{c}5.73 \pm 1.86 \\
(0.96-12.42)\end{array}$ & $\begin{array}{c}5.93 \pm 1.55 \\
(1.86-11.23)\end{array}$ & $\begin{array}{c}9.13 \pm 1.57 \\
(5.51-12.40)\end{array}$ & 0.40 & $<0.01$ \\
\hline Median left lung dose/Gy & $\begin{array}{c}1.48 \pm 0.48 \\
(0.43-3.63)\end{array}$ & $\begin{array}{c}1.48 \pm 0.48 \\
(0.68-3.47)\end{array}$ & $\begin{array}{c}1.96 \pm 0.63 \\
(1.11-3.53)\end{array}$ & 0.99 & $<0.01$ \\
\hline Left Lung V20/cc & $\begin{array}{c}97.5 \pm 50.7 \\
(0.8-269.8)\end{array}$ & $\begin{array}{c}171.1 \pm 63.7 \\
(25.5-349.8)\end{array}$ & $\begin{array}{c}321.3 \pm 76.6 \\
(105.9-434.8)\end{array}$ & $<0.01$ & $<0.01$ \\
\hline Left lung V20/\% & $\begin{array}{c}8.8 \pm 3.7 \\
(0.1-22.6)\end{array}$ & $\begin{array}{c}9.3 \pm 3.2 \\
(1.3-20.4)\end{array}$ & $\begin{array}{c}16.9 \pm 3.4 \\
(9.1-24.2)\end{array}$ & 0.30 & $<0.01$ \\
\hline
\end{tabular}

cc: cubic centimeter. The p-values are from unpaired t-tests between two patient groups. A - B: The p-value for t-test between Groups A and B; A - C: The p-value for t-test between Groups A and C.

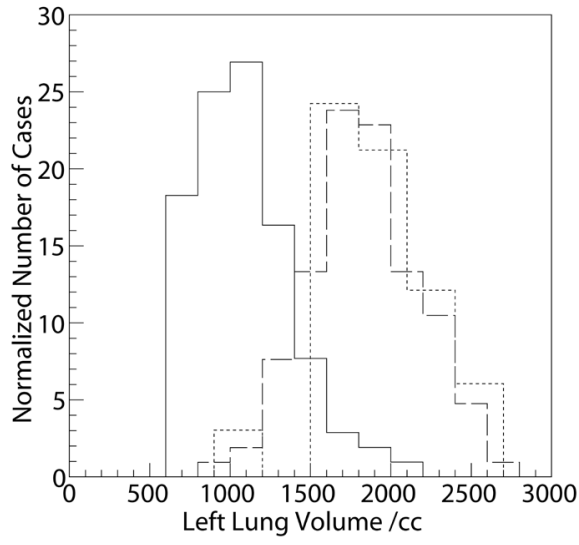

(a)

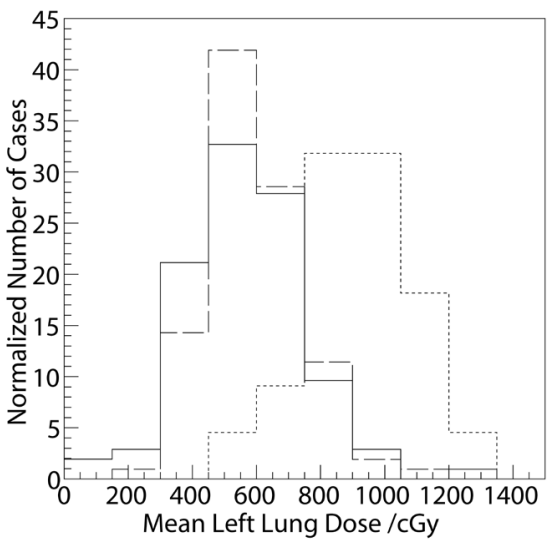

(b)

Figure 3. Left lung volume (a) and the mean left lung dose (b) distributions in the three groups: Group A (solid lines), Group B (dashed lines), and Group C (dotted lines). 
group. Despite significant increase in left lung volume, the average mean left lung dose in Group B was $5.93 \pm 1.55$ Gy, slightly higher than the average mean left lung dose of $5.73 \pm 1.86 \mathrm{~Gy}$ in Group A with no statistical significance. On the other hand, the average mean left lung dose was $9.13 \pm 1.57$ Gy in Group C, significantly higher than those in Groups A and B due to the use of wide tangential fields. Figure 3(b) shows normalized mean left lung dose distributions in each patient group. There was no statistically significant difference in relative left lung V20 between Groups A and B: $8.8 \% \pm 3.7 \%$ in Group A and 9.3\% $\pm 3.2 \%$ in Group B.

\section{Discussions}

In the US, one out of eight women will be diagnosed with breast cancer in her lifetime [16]. Adjuvant post-operative RT can significantly reduce disease recurrence rate but is associated with an increase in mortality due to hear disease and lung cancer, potentially negating the cancer-specific survival benefit [2] [5] [6]. This study used longitudinal clinical data to evaluate clinically achievable reduction of heart dose and ischemic heart disease risks with the implementation of the DIBH technique, as well as the clinical impact on left lung dose. Compared to pre-clinical dosimetric evaluation studies on the DIBH technique in the literature, this study demonstrated clinically achieved benefits of the DIBH technique with clinical data over a six-year period.

Among the different endpoints reported in published studies on radiation-induced heart toxicities, ischemic heart disease is responsible for increased mortality [11]. Based on normal tissue complication probability (NTCP) modeling, heart V25 was estimated to correlate with cardiac mortality [17] [18]. This study found that when the DIBH technique was used, heart V25 was low even for patients treated with wide tangential fields for IMN (Table 2), indicating minimal cardiac mortality based on NTCP modeling. Darby et al. carried out a population-based study in 2168 patients and found that the increased risk of ischemic heart diseased increased linearly with the mean dose to the heart, with no apparent threshold [11]. Based on this linear model, the increased risk for ischemic heart disease for patients using the DIBH technique was less than half of that for patients treated without the DIBH technique (Table 2). This risk reduction will have significant long-term benefit to breast cancer patients.

In post-operative external-beam RT of breast cancer, acute effects to the lung include radiation pneumonitis, while chronic and late effects include tissue fibrosis and radiation-induced secondary lung cancer [6] [7]. Mild and moderate radiation pneumonitis occurs in up to $20 \%$ of patients, although severe radiation pneumonitis seldom happens [7]. Since the lung is one of the organs that are most sensitive to radiation-induced secondary cancer risks [19] [20], an increased lung cancer rate may offset survival benefits from post-operative RT for breast cancer patients [6]. It is therefore imperative to limit lung dose during RT in the thoracic region. The lung V20 and mean lung dose (MLD) are two most commonly used dosimetric parameters in evaluating lung toxicity risks [21]. 
Since the right lung volume is typically larger than the left lung volume, the total lung V20 and the total MLD are expected to be less than half of those for the left lung as presented for each patient group in Table 3. As shown in Table 3, there was no significant difference in either relative V20 for the left lung or the mean left lung dose between Groups A and B. Based on secondary lung cancer occurrence models [22] [23] [24], using the DIBH technique would not significantly increase lung cancer risks during external-beam RT for left-sided breast cancer.

Irradiation of IMN nodes has been a controversial practice in post-operative radiotherapy of breast cancer as the rate of IMN recurrence is low and there is concern of increased cardiac and pulmonary toxicities. As recent studies demonstrated survival benefits of regional lymph node irradiation [25] [26], the National Comprehensive Cancer Network (NCCN) Breast Cancer Clinical Practice Guidelines raised the level of recommendation for IMN irradiation in 2016 [27]. As a result, more breast cancer patients receive IMN irradiation in post-operative radiotherapy in recent years. Since a larger normal tissue volume is irradiated with wide tangent fields, the use of the DIBH technique could be especially beneficialin sparing critical organs when wide tangent fields are used to cover the IMN [28] [29]. In our patient group, the inclusion of IMN in tangential field treatments with the DIBH technique still gave a $52.6 \%$ reduction in the average mean heart dose compared to patients treated without the DIBH technique. Despite the use of wide tangential fields, the total MLD and total lung V20 for Group C patients were estimated to be well within recommended dose limits [21] [30] [31].

Respiratory gating in RT is now a mature technology. Besides using marker blocks in the abdominal region, the DIBH technique can also be implemented with the surface-guided radiotherapy (SGRT) technique [32] [33]. Based on our clinical experience, the respiratory gating technique can be implemented in the clinic without requiring excessive resource and staffing. Given the significant benefits in heart toxicity reduction, the DIBH technique is recommended in post-operative external-beam RT of left-sided breast cancer.

\section{Conclusion}

Based on longitudinal clinical data from a single institution, this study showed that implementation of the DIBH technique significantly lowered heart dose and decreased ischemic heart disease risk. The DIBH technique did not significantly increase lung toxicity when the IMN was not irradiated. The DIBH technique is recommended in post-operative external-beam radiotherapy of left-sided breast cancer.

\section{Conflicts of Interest}

None.

\section{References}

[1] Siegel, R.L., Miller, K.D. and Jemal, A. (2019) Cancer Statistics, 2019. CA: A Cancer 
Journal for Clinicians, 69, 7-34. https://doi.org/10.3322/caac.21551

[2] Early Breast Cancer Trialists' Collaborative Group (EBCTCG) (2011) Effect of Radiotherapy after Breast-Conserving Surgery on 10-Year Recurrence and 15-Year Breast Cancer Death: Meta-Analysis of Individual Patient Data for 10801 Women in 17 Randomised Trials. The Lancet, 378, 1707-1716. https://doi.org/10.1016/S0140-6736(11)61629-2

[3] Fisher, B., Anderson, S., Redmond, C.K., et al. (1995) Reanalysis and Results after 12 Years of Follow-Up in a Randomized Clinical Trial Comparing Total Mastectomy with Lumpectomy with or without Irradiation in the Treatment of Breast Cancer. The New England Journal of Medicine, 333, 1456-1461. https://doi.org/10.1056/NEJM199511303332203

[4] Polgár, C., Limbergen, E.V., Pötter, R., et al. (2010) Patient Selection for Accelerated Partial-Breast Irradiation (APBI) after Breast-Conserving Surgery: Recommendations of the Groups Eurpeen de Curietherapie-European Society for Therapeutic Radiology and Oncology (GEC-ESTRO) Breast Cancer Working Group Based on Clinical Evidence. Radiotherapy and Oncology, 94, 264-273. https://doi.org/10.1016/j.radonc.2010.01.014

[5] Whelan, T.J., Julian, J., Wright, J., et al. (2000) Does Locoregional Radiation Therapy Improve Survival in Breast Cancer? A Meta-Analysis. Journal of Clinical Oncology, 18, 1220-1229. https://doi.org/10.1200/JCO.2000.18.6.1220

[6] Early Breast Cancer Trialists' Collaborative Group (EBCTCG) (2005) Effect of Radiotherapy and Differences in the Extent of Surgery for Early Breast Cancer on Local Recurrence and 15-Year Survival: An Overview of the Randomized Trials. The Lancet, 366, 2087-2106. https://doi.org/10.1016/S0140-6736(05)67887-7

[7] Marks, L.B., Yu, X., Vujaskovic, V., et al. (2003) Radiation-Induced Lung Injury. Seminars in Radiation Oncology, 13, 333-345.

https://doi.org/10.1016/S1053-4296(03)00034-1

[8] Kole, A.J., Kole, L. and Moran, M.S. (2017) Acute Radiation Dermatitis in Breast Cancer Patients: Challenges and Solutions. Breast Cancer-Targets and Therapy, 9, 313-323. https://doi.org/10.2147/BCTT.S109763

[9] Harris, S.R. (2016) Differentiating the Causes of Spontaneous Rib Fracture after Breast Cancer. Clinical Breast Cancer, 16, 431-436. https://doi.org/10.1016/j.clbc.2016.07.001

[10] Pierce, S.M., Recht, A., Lingos, T.I., et al. (1992) Long-Term Radiation Complications Following Conservative Surgery (CS) and Radiation Therapy (RT) in Patients with Early Stage Breast Cancer. International Journal of Radiation Oncology, Biology, Physics, 23, 915-923. https://doi.org/10.1016/0360-3016(92)90895-O

[11] Darby, S.C., Ewertz, M., McGale, P., et al. (2013) Risk of Ischemic Heart Disease in Women after Radiotherapy for Breast Cancer. The New England Journal of Medicine, 368, 987-998. https://doi.org/10.1056/NEJMoa1209825

[12] Mast, M.E., Kempen-Harteveld, L., Heijenbrok, M.W., et al. (2013) Left-Sided Breast Cancer Radiotherapy with and without Breath-Hold: Does IMRT Reduce the Cardiac Dose Even Further? Radiotherapy and Oncology, 108, 248-253. https://doi.org/10.1016/j.radonc.2013.07.017

[13] Hayden, A.J., Rains, M. and Tiver, K. (2012) Deep Inspiration Breath Hold Technique Reduces Heart Dose from Radiotherapy for Left-Sided Breast Cancer. Journal of Medical Imaging and Radiation Oncology, 56, 464-472. https://doi.org/10.1111/j.1754-9485.2012.02405.x

[14] Pedersen, A.N., Korreman, S., Nyström, H., et al. (2004) Breathing Adapted Radio- 
therapy of Breast Cancer: Reduction of Cardiac and Pulmonary Doses Using Voluntary Inspiration Breath-Hold. Radiotherapy and Oncology, 72, 53-60. https://doi.org/10.1016/j.radonc.2004.03.012

[15] Remouchamps, V.M., Vicini, F.A., Sharpe, M.B., et al. (2003) Significant Reductions in Heart and Lung Doses Using Deep Inspiration Breath Hold with Active Breathing Control and Intensity-Modulated Radiation Therapy for Patients Treated with Locoregional Breast Irradiation. International Journal of Radiation Oncology, Biology, Physics, 55, 392-406. https://doi.org/10.1016/S0360-3016(02)04143-3

[16] Feur, E.J., Wun, L., Boring, C.C., et al. (1993) The Lifetime Risk of Developing Breast Cancer. Journal of the National Cancer Institute, 85, 892-897. https://doi.org/10.1093/jnci/85.11.892

[17] Gagliardi, G., Constine, L.S., Moiseenko, V., et al. (2010) Radiation Dose-Volume Effects in the Heart. International Journal of Radiation Oncology, Biology, Physics, 76, S77-S85. https://doi.org/10.1016/j.ijrobp.2009.04.093

[18] Moiseenko, V., Einck, J., Murphy, J., et al. (2016) Clinical Evaluation of QUANTEC Guidelines to Predict the Risk of Cardiac Mortality in Breast Cancer Patients. Acta Oncologica, 55, 1506-1510. https://doi.org/10.1080/0284186X.2016.1234067

[19] Hancock, S.L. and Hoppe, R.T. (1996) Long-Term Complications of Treatment and Causes of Mortality after Hodgkin's Disease. Seminars in Radiation Oncology, 6, 225-242. https://doi.org/10.1016/S1053-4296(96)80018-X

[20] Boice, J.D., Preston, D., Davis, F.G., et al. (1991) Frequent Chest X-Ray Fluoroscopy and Breast Cancer Incidence among Tuberculosis Patients in Massachusetts. Radiation Research, 125, 214-222. https://doi.org/10.2307/3577890

[21] Marks, L.B., Bentzen, S.M., Deasy, J.O., et al. (2010) Radiation Dose-Volume Effects in the Lung. International Journal of Radiation Oncology, Biology, Physics, 76, S70-S76. https://doi.org/10.1016/j.ijrobp.2009.06.091

[22] Schneider, U., Zwahlen, D., Ross, D. and Kaser-Hotz, B. (2005) Estimation of Radiation-Induced Cancer from Three-Dimensional Dose Distributions: Concept of Organ Equivalent Dose. International Journal of Radiation Oncology, Biology, Physics, 61, 1510-1515. https://doi.org/10.1016/j.ijrobp.2004.12.040

[23] Abo-Madyan, Y., Aziz, M.H., Aly, M., et al. (2014) Second Cancer Risk after 3D-CRT, IMRT and VMAT for Breast Cancer. Radiotherapy and Oncology, 110, 471-476. https://doi.org/10.1016/j.radonc.2013.12.002

[24] Han, C., Schultheiss, E.T. and Wong, J.Y.C. (2017) Estimation of Radiation-Induced Secondary Cancer Risks for Early-Stage Non-Small Cell Lung Cancer Patients after Stereotactic Body Radiation Therapy. Practical Radiation Oncology, 7, e185-e194. https://doi.org/10.1016/j.prro.2016.10.009

[25] Whelan, T.J., Olivotto, I.A., Parulekar, W.R., et al. (2015) Regional Nodal Irradiation in Early-Stage Breast Cancer. The New England Journal of Medicine, 373, 307-316. https://doi.org/10.1056/NEJMoa1415340

[26] Poortmans, P.M., Collette, S., Kirkove, C., Van Limbergen, E., et al. (2015) Internal Mammary and Medial Supraclavicular Irradiation in Breast Cancer. The New England Journal of Medicine, 373, 317-327. https://doi.org/10.1056/NEJMoa1415369

[27] Gradishar, W. and Salerno, K.E. (2016) NCCN Guidelines Update: Breast Cancer. Journal of the National Comprehensive Cancer Network, 14, 641-644. https://doi.org/10.6004/jnccn.2016.0181

[28] Hjelstuen, M.B., Mjaaland, I., Vikström, J. and Dybvik, K.I. (2012) Radiation during Deep Inspiration Allows Loco-Regional Treatment of Left Breast and Axillary-, Su- 
praclavicular- and Internal Mammary Lymph Nodes without Compromising Target Coverage or Dose Restrictions to Organs at Risk. Acta Oncologica, 51, 333-344. https://doi.org/10.3109/0284186X.2011.618510

[29] Stranzl, H., Zurl, B., Langsenlehner, T. and Kapp, K.S. (2009) Wide Tangential Fields Including the Internal Mammary Lymph Nodes in Patients with Left-Sided Breast Cancer. Strahlentherapie und Onkologie, 185, 155-160. https://doi.org/10.1007/s00066-009-1939-2

[30] Kwa, S.L., Lebesque, J.V., Theuws, J.C.M., et al. (1998) Radiation Pneumonitis as a Function of Mean Lung Dose: An Analysis of Pooled Data of 540 Patients. International Journal of Radiation Oncology, Biology, Physics, 43, 1-9. https://doi.org/10.1016/S0360-3016(98)00196-5

[31] Mamounas, E.P., Bandos, H., White, J.R., et al. (2018) NRG Oncology/NSABP B-51/RTOG 1304: Phase III Trial to Determine If Chest Wall and Regional Nodal Radiotherapy (CWRNRT) Post Mastectomy (Mx) or the Addition of RNRT to Breast RT Post Breast-Conserving Surgery (BCS) Reduces Invasive Breast Cancer Recurrence-Free Interval (IBCR-FI) in Patients (pts) with Positive Axillary (PAx) Nodes Who Are ypN0 after Neoadjuvant Chemotherapy (NC). Journal of Clinical Oncology, 36, TPS601-TPS601. https://doi.org/10.1200/JCO.2018.36.15_suppl.TPS601

[32] Cerviño, L.I., Gupta, S., Rose, M.A., et al. (2009) Using Surface Imaging and Visual Coaching to Improve the Reproducibility and Stability of Deep-Inspiration Breath Hold for Left-Breast-Cancer Radiotherapy. Physics in Medicine \& Biology, 54, 6853-6865. https://doi.org/10.1088/0031-9155/54/22/007

[33] Betgen, A., Alderliesten, T., Sonke, J., et al. (2013) Assessment of Set-Up Variability during Deep Inspiration Breath Hold Radiotherapy for Breast Cancer Patients by 3D-Surface Imaging. Radiotherapy and Oncology, 106, 225-230.

https://doi.org/10.1016/j.radonc.2012.12.016 